\title{
Álgebra e teoria dos números para olimpíadas
}

\author{
Ana P. Chaves
}

\section{Resumo}

No texto a seguir, iremos nos debruçar sobre problemas de várias olimpíadas de Matemática, tanto nacionais como internacionais, com foco nos que abordam temas de álgebra e Teoria dos Números. O objetivo principal é estimular o envolvimento de alunos e professores em tais competições, discutindo soluções e propondo problemas.

Palavras-chave: Olimpíadas de matemática; álgebra; teoria dos números.

\section{Olimpíadas: Um pouco de história}

As Olimpíadas de Matemática, como conhecemos atualmente, são disputadas desde 1894, quando foram organizadas algumas competições na Hungria. A partir daí, competições similares espalharamse pelo leste europeu, culminando, em 1959, com a organização da $1^{a}$ Olimpíada Internacional de Matematica (IMO), na Romênia, com a participação de apenas 7 países daquela região. Deste então, a competição tem se expandido, e em 2017, pela primeira vez realizada no Brasil, a $58^{a}$ a IMO contou com a participação de 623 estudantes de 112 países de cinco continentes.

No Brasil, a Sociedade Brasileira de Matemática (SBM) organiza desde 1979 a Olimpíada Brasileira de Matemática (OBM), chegando hoje à sua 41ํㅡ edição. Ao longo dos anos, várias olimpíadas regionais surgiram, entre elas, a Olimpíada de Matematica do Estado de Goiás (Omeg), realizada desde 1992 pelo IME-UFG. A ideia central dessas competições é estimular o estudo da Matemática pelos alunos, desenvolver e aperfeiçoar a capacitação dos professores, influenciar na melhoria do ensino, além de descobrir jovens talentos.

Vários ex-medalhistas da IMO, como Artur Ávila, Grigory Margulis, Jean-Christophe Yoccoz, Laurent Lafforgue, Stanislav Smirnov, Terence Tao, Su-charit Sarkar, Grigori Perelman, Ngo Bao Chau e Maryam Mirzakhani, tornaram-se matemáticos exepcionais, sendo premiados com a medalha Fields, prêmio máximo da Matemática mundial.

\section{Problemas de álgebra}

A álgebra tem um papel fundamental, não apenas na Matemática, mas também em outros vários ramos da ciência. Sem a álgebra, não haveria uma linguagem uniforme para se expressar conceitos como a aritmética ou propriedades dos números. Portanto, é de extrema importância conhecer bem essa linguagem para ser capaz de compreender bem outras áreas da Matemática, tais como Teoria dos Números, Combinatória e até Geometria. Os problemas a seguir discutirão técnicas importantes que também são aplicáveis a outros tantos problemas de Olimpíada. 


\subsection{Problema 1}

(OBM 2010) Sejam $a, b$ e $c$ números reais tais que $a \neq b$ e $a^{2}(b+c)=b^{2}(c+a)=2010$. Calcule $c^{2}(a+b)$.

Solução: Primeiro, note que de $a^{2}(b+c)=b^{2}(c+a)$, obtemos

$$
\begin{aligned}
a^{2}(b+c) & =b^{2}(c+a) \\
a^{2} b-b^{2} a+a^{c}-b^{c} & =0 \\
a b(a-b)+c(a+b)(a-b) & =0 \\
(a-b)(a b+b c+c a) & =0 .
\end{aligned}
$$

Como $a \neq b$, a última igualdade dá-nos $a b+b c+c a=0$. Agora, usando que $b^{2}(c+a)=2010$, tomando o valor desejado $c^{2}(a+b)=k$ e subtraindo ambos, conseguimos

$$
\begin{aligned}
2010-k & =b^{2} c-c^{2} b+b^{2} a-c^{2} a \\
& =b c(b-c)+a(b+c)(b-c) \\
& =(b-c)(a b+b c+c a) .
\end{aligned}
$$

Mas como já havíamos conseguido $a b+b c+c a=0$, isso nos dá que o lado esquerdo da última igualdade acima é igual a zero, donde $c^{2}(a+b)=k=2010$.

\subsection{Problema 2}

(Romênia 2006) Encontre todos os números reais $a$ e $b$ satisfazendo

$$
2\left(a^{2}+1\right)\left(b^{2}+1\right)=(a+1)(b+1)(a b+1) .
$$

Solução: Usando produtos notáveis, vamos desenvolver a equação dada no problema:

$$
\begin{aligned}
2\left(a^{2}+1\right)\left(b^{2}+1\right)= & (a+1)(b+1)(a b+1) \\
2\left(a^{2} b^{2}+a^{2}+b^{2}+1\right)= & (a b+a+b+1)(a b+1) \\
2 a^{2} b^{2}+2 a^{2}+2 b^{2}+2= & a^{2} b^{2}+a^{2} b+a b^{2}+2 a b \\
& +a+b+1,
\end{aligned}
$$

e isolando a no lado esquerdo da última igualdade, dá-nos

$$
a^{2}\left(b^{2}-b+2\right)-a\left(b^{2}+2 b+1\right)+2 b^{2}-b+1=0,
$$

que podemos interpretar como uma equação do $2^{o}$ em $a$, cujo discriminante $(\Delta)$ é:

$$
\begin{aligned}
\Delta & =(b+1)^{4}-4\left(b^{2}-b+2\right)\left(2 b^{2}-b+1\right) \\
& =-7 b^{4}+16 b^{3}-18 b^{2}+16 b-7 .
\end{aligned}
$$


Esse polinômio possui duas características interessantes. A primeira, que nós não vamos utilizar, é que ele é um polinômio recíproco de $4^{o} \underline{o}$ grau e $1^{\underline{a}}$ espécie, já que a leitura dos seus coeficientes feita da esquerda para a direita coincide com a leitura feita da direita para a esquerda. A segunda é que $b=1$ é uma raiz desse polinômio, já que a soma dos seus coeficientes é igual a 0. Isso nos dá

$$
\begin{aligned}
\Delta & =-7 b^{4}+7 b^{3}+9 b^{3}-9 b^{2}-9 b^{2}+9 b+7 b-7 \\
& =-7 b^{3}(b-1)+9 b^{2}(b-1)-9 b(b-1)+7(b-1) \\
& =(b-1)\left(-7 b^{3}+9 b^{2}-9 b+7\right) .
\end{aligned}
$$

Novamente, o segundo fator desse último produto tem a soma dos seus coeficientes igual a zero, donde $b=1$ também é raiz do mesmo, e escrevendo $-7 b^{3}+9 b^{2}-9 b+7=(b-1)\left(-7 b^{2}+2 b-7\right)$, obtemos

$$
\Delta=(b-1)^{2}\left(-7 b^{2}+2 b-7\right) .
$$

O discriminante de $-7 b^{2}+2 b-7$ é negativo e, portanto, $-7 b^{2}+2 b-7<0, \forall b$. Como $(b-1)^{2} \geq 0$, $\forall b$, segue que $\Delta \leq 0, \forall b$. Para $a \in \mathbb{R}$, devemos então ter $\Delta=0$ e, portanto, $b=1$ e $a=1$, que é a única solução.

\subsection{Problema 3}

(OMEG 2017) Encontre todos os números naturais $x$ tais que o produto dos seus dígitos (em notação decimal) é igual a $x^{2}-10 x-22$.

Solução: Seja $x \in \mathbb{N}$ escrito como $x=\overline{d_{1} d_{2} \ldots d_{k}}$ na base 10 . Sabemos que os dígitos $d_{i} \in$ $\left\{0,1,2, \ldots, 9\right.$, para todo $i=1,2, \ldots, k$, com $d_{1} \neq 0$. Sendo assim, temos que, denotando o produto dos dígitos por $P=d_{1} \cdot d_{2} \cdots d_{k}$, as seguintes desigualdades são válidas:

$$
x=\overline{d_{1} d_{2} \ldots d_{k}} \geq 10^{k}>d_{1} \cdot d_{2} \cdots d_{k}=P .
$$

Assim, obtemos

$$
x>P=x^{2}-10 x-22 \Rightarrow x^{2}-11 x-22<0 .
$$

Agora, observe que as únicas soluções naturais são $\{1,2, \ldots, 12\}$, e como o único desses números que satisfaz $P=x^{2}-10 x-22$, é

$$
12^{2}-10 \cdot 12-22=2=1 \cdot 2,
$$

donde concluímos que $x=12$ é a única solução.

\subsection{Problema 4}

(Bulgária 1997) Encontre todos os naturais $a, b, c$ tais que as raízes das equações

$$
\begin{aligned}
& x^{2}-2 a x+b=0 \\
& x^{2}-2 b x+c=0 \\
& x^{2}-2 c x+a=0
\end{aligned}
$$


Solução: Sejam $\left(x_{1}, x_{2}\right),\left(x_{3}, x_{4}\right)$ e $\left(x_{5}, x_{6}\right)$ respectivamente as raízes das três equações dadas. Então, pelas relações de soma e produto, temos que:

$$
\text { (I) } \begin{aligned}
x_{1}+x_{2} & =2 a \\
x_{1} x_{2} & =b
\end{aligned}
$$

(II) $\begin{aligned} x_{3}+x_{4} & =2 b \\ x_{3} x_{4} & =c\end{aligned}$

(III) $\begin{aligned} x_{5}+x_{6} & =2 c \\ x_{5} x_{6} & =a\end{aligned}$.

Suponha que todas essas raízes são pelo menos iguais a 2 , i.e., $x_{i} \geq 2, \forall i$. Então,

$$
\begin{aligned}
& 2 a=x_{1}+x_{2} \leq x_{1} x_{2}=b \\
& 2 b=x_{3}+x_{4} \leq x_{3} x_{4}=c \\
& 2 c=x_{5}+x_{6} \leq x_{5} x_{6}=a
\end{aligned}
$$

donde somando as pontas das três desigualdades, obtemos $2(a+b+c) \leq a+b+c$, o que é impossível já que $a, b, c \in \mathbb{N}$. Portanto, pelo menos uma das raízes deve ser igual a 1 . Supondo, sem perda de generalidade, que $x_{1}=1$, temos por $(\mathrm{I})$ :

$$
\begin{aligned}
1+x_{2} & =2 a \\
1 \cdot x_{2} & =b
\end{aligned} \Rightarrow 2 a-b=1 \text {. (IV) }
$$

Agora, suponha $x_{i} \geq 2$, para $i=2,3,4,5,6$. Então, de (II) e (III), conseguimos

$$
2 b+2 c=\left(x_{3}+x_{4}\right)+\left(x_{5}+x_{6}\right) \leq x_{3} x_{4}+x_{5} x_{6}=c+a \Rightarrow 2(2 a-1)+2 c \leq c+a,
$$

donde $c \leq 2-3 a$, o que é um absurdo para $a, c \in \mathbb{N}$. Portanto, pelo menos uma das raízes $x_{3}, x_{4}, x_{5}$ ou $x_{6}$ é igual a 1 . Novamente, s.p.g., tome $x_{3}=1$. Análogo a (IV), temos $2 b-c=1$ (V). Usando novamente o mesmo argumento, suponha $x_{5}, x_{6} \geq 2$,

$$
2 c \leq a \Rightarrow 2 \overbrace{(2 b-1)}^{(\mathrm{V})} \leq \overbrace{\frac{b+1}{2}}^{(\mathrm{IV})} \Rightarrow 7 b \leq 5 . \text { Absurdo! }
$$

Portanto, uma das raízes, $x_{5}$ ou $x_{6}$, é igual a 1 , donde segue $2 c-a=1$ (VI). Finalmente, por (IV)-(VI), obtemos,

$$
(2 a-b-1)+(2 b-c-1)+(2 c-a-1)=0 \Rightarrow a+b+c=3
$$

e como $a, b$ e $c$ são naturais, a única solução para a igualdade acima é $a=b=c=1$.

\subsection{Problema 5}

[4] (Vietnã 2008) Sejam $x, y$ e $z$ reais distintos não negativos. Mostre que

$$
(x y+y z+z x)\left(\frac{1}{(x-y)^{2}}+\frac{1}{(y-z)^{2}}+\frac{1}{(z-x)^{2}}\right) \geq 4 .
$$

\section{Quando ocorre a igualdade?}


Solução: Sem perda de generalidade, podemos supor que $z>y>x \geq 0$, já que são todos distintos e não negativos. Assim, defina $y=x+a, z=x+a+b$, com $a, b>0$, e substituindo do lado esquerdo da desigualdade, dá-nos

$$
M=\left[3 x^{2}+2(2 a+b) x+a(a+b)\right]\left(\frac{1}{a^{2}}+\frac{1}{b^{2}}+\frac{1}{(a+b)^{2}}\right) .
$$

Então, temos que

$$
\begin{aligned}
M & \geq a(a+b)\left(\frac{1}{a^{2}}+\frac{1}{b^{2}}+\frac{1}{(a+b)^{2}}\right) \\
& =\frac{a+b}{a}+\frac{a(a+b)}{b^{2}}+\frac{a}{a+b} \\
& =1+\frac{b}{a}+\frac{a(a+b)}{b^{2}}+1-\frac{b}{a+b} \\
& =2+\frac{a(a+b)}{b^{2}}+\frac{b^{2}}{a(a+b)} \\
& =2+\frac{[a(a+b)]^{2}+\left(b^{2}\right)^{2}}{b^{2} a(a+b)} \geq 2+2=4 .
\end{aligned}
$$

A igualdade ocorre se, e somente se, temos

$$
\left\{\begin{aligned}
3 x^{2}+2(2 a+b) x & =0 \\
a(a+b) & =b^{2}
\end{aligned}\right.
$$

que nos dá $x=0$ e $a(a+b)=b^{2}$. Nesse caso, temos $y=a$ e $z=a+b$, donde $b=z-y$. Portanto, para $a(a+b)=b^{2}$, temos $y z=(z-y)^{2}$, dando-nos $y^{2}-3 y z+z^{2}=0$. Fazendo $t=y / z \in(0,1)$, a equação anterior se reescreve como $t^{2}-3 t+1$, daí $t=\frac{3-\sqrt{5}}{2}$. Assim, $x=0$ e $\frac{y}{z}=\frac{3-\sqrt{5}}{2}$.

\subsection{Problemas Complementares}

$[3,5,6]$

1. (OCM) Prove que não existem inteiros positivos $a$ e $b$ tais que $\frac{b^{2}+b}{a^{2}+a}=4$.

2. Seja $D=a^{2}+b^{2}+c^{2}$, sendo $a, b$ e $c$ inteiros consecutivos e $c=a b$. Mostre que $\sqrt{D}$ é sempre um inteiro ímpar.

3. (IMO) Mostre que

$$
\frac{a}{\sqrt{a^{2}+8 b c}}+\frac{b}{\sqrt{b^{2}+8 c a}}+\frac{c}{\sqrt{c^{2}+8 a b}} \geq 1
$$

para todos $a, b$ e $c$ reais positivos.

4. (AIME) Seja $f(x)$ um polinômio com coeficientes reais tal que $f(0)=1, f(2)+f(3)=125$, e para todo $x, f(x) f\left(2 x^{2}\right)=f\left(2 x^{3}+x\right)$. Encontre $f(5)$. 
5. (USAMO) Sejam $a, b$ e $c$ reais positivos. Prove que

$$
\left(a^{5}-a^{2}+3\right)\left(b^{5}-b^{2}+3\right)\left(c^{5}-c^{2}+3\right) \geq(a+b+c)^{3} .
$$

6. (INAMO) Determine todos os pares distintos de números reais $(x, y)$ tais que ambas as identidades são verdadeiras:

$$
\begin{aligned}
& x^{100}-y^{100}=2^{99}(x-y) \\
& x^{200}-y^{200}=2^{199}(x-y) .
\end{aligned}
$$

7. (JBMO) Sejam $x$ e $y$ reais positivos tais que

$$
x^{3}+y^{3}+(x+y)^{3}+30 x y=2000 .
$$

Mostre que $x+y=10$.

8. (CMO) Encontre todos os trios $(x, y, z)$ tais que quando qualquer um desses números é adicionado ao produto dos outros dois, o resultado é igual a 2.

9. (IMTT) Prove que a equação

$$
x^{2}+y^{2}-z^{2}=1997
$$

tem infinitas soluções inteiras $(x, y, z)$.

10. (USAMO) Encontre todas as soluções naturais da equação

$$
a^{2}+b^{2}+c^{2}=a^{2} b^{2} .
$$

11. (HMO) Sejam $x, y, z$ reais distintos dois a dois, tais que

$$
\frac{x}{y-z}+\frac{y}{z-x}+\frac{z}{x-y}=0
$$

Prove que

$$
\frac{x}{(y-z)^{2}}+\frac{y}{(z-x)^{2}}+\frac{z}{(x-y)^{2}}=0 .
$$




\section{Problemas de Teoria dos Números}

Teoria dos Números é uma das áreas mais antigas da Matemática. é onde se encontram inúmeras conjecturas, a priori simples, mas que estiveram sem solução por séculos, e a Teoria dos Números continua expandindo as fronteiras da pesquisa até hoje. Mesmo com a quantidade de problemas resolvidos aumentando, também aumentam a quantidade de problemas em aberto, que podem demorar anos e muito esforço para serem superados. Nos dias de hoje, a "demanda" dessa área também cresceu em aplicações, tais como na Criptografia e na Ciência da Computação.

Como sugere o nome, Teoria dos Números é o estudo dos números e suas propriedades. Uma parte importante dessa vasta área lida com soluções inteiras de equações, ditas Equações Diofantinas (em homenagem ao matemático grego Diofantus), com algumas consequências bastante interessantes sobre números primos e aritmética. Devido à sua natureza fundamentalmente algébrica, as técnicas da Teoria dos Números dependem fortemente da "maquinária algébrica", donde existe uma considerável interseção entre as duas áreas.

Os tópicos de Teoria dos Números tratados nos problemas a seguir são elementares, com foco principalmente nas estratégias fundamentais que aparecem com frequência em problemas de níveis iniciante e intermediário de Olimpíada. Todos o background necessário para a solução dos problemas desta seção, pode ser encontrado nas excelentes referências [1, 2, 7, 8].

\subsection{Problema 1}

(IMO 1975) Seja $S(n)$ a soma dos dígitos de $n$. Se $N=4444^{4444}, A=S(N)$ e $B=S(A)$. Quanto vale $S(B)$ ?

Solução: Pelo critério de divisibilidade por 9 , sabemos que $N \equiv A \equiv B(\bmod 9)$. Primeiro, vamos calcular o resto de $N$ por 9 . Como $4444 \equiv 16 \equiv 7(\bmod 9)$, então $4444^{4444} \equiv 7^{4444}(\bmod 9)$, donde precisamos encontrar o resto de $7^{4444}$. Note que, $7^{2} \equiv 49 \equiv 4(\bmod 9) \Rightarrow 7^{3} \equiv 28 \equiv 1(\bmod 9)$, donde

$$
\begin{gathered}
7^{4444}=7^{3 \cdot 1481+1}=\left(7^{3}\right)^{1481} \cdot 7 \equiv 7(\bmod 9), \\
\Rightarrow 7^{4444} \equiv 7(\bmod 9) .
\end{gathered}
$$

Por outro lado, como $N=4444^{4444}<10^{5 \cdot 4444}$, então $S(N)<5 \cdot 4444 \cdot 9=199980$. Além disso, $B=S(A) \leq 1+9 \cdot 5=46$ e $S(B) \leq 12$. O único inteiro menor ou igual a 12 com resto 7 por 9 , é o próprio 7 , daí $S(B)=7$.

\subsection{Problema 2}

(Estônia 2000) Prove que não é possível dividir qualquer conjunto de 18 inteiros consecutivos em dois conjuntos disjuntos $A$ e $B$ tais que o produto dos elementos de $A$ seja igual ao produto dos elementos de $B$ 
Solução: Suponha, por absurdo, que existam tais conjuntos, e que os 18 consecutivos são $n, n+$ $1, \ldots, n+17$. Agora, note que, como o produto dos elementos de $A$ é igual ao produto dos elementos de $B$, se um dos conjuntos contém um múltiplo de 19, o outro também deve conter. Entre 18 inteiros consecutivos, porém, não existem dois múltiplos de 19. Desse modo, nenhum dos dois conjuntos possui elementos divisíveis por 19. Denote por $r$ o resto da divisão do produto de todos os elementos de $A, P_{A}$, por 19 , que é o mesmo do produto dos elementos de $B, P_{B}$, já que supomos que são iguais. Assim,

$$
\begin{aligned}
r \cdot r=P_{A} \cdot P_{B} & \equiv n(n+1)(n+2) \cdots(n+17) \quad(\bmod 19) \\
& \equiv 1 \cdot 2 \cdot 3 \cdots 18 \quad(\bmod 19) \\
& \equiv(19-1) !(\bmod 19) \\
& \equiv-1 \quad(\bmod 19) \\
\Rightarrow r^{2} & \equiv-1 \quad(\bmod 19)
\end{aligned}
$$

onde (1) vem do fato de o conjunto de 18 elementos consecutivos não possuir múltiplos de 19, e (2) é consequência do Teorema de Wilson. Assim, $r^{2} \equiv-1(\bmod 19) \Rightarrow r^{18} \equiv(-1)^{9} \equiv-1(\bmod 19)$, mas isso contraria o Pequeno Teorema de Fermat e conseguimos um absurdo. Portanto, tais conjuntos não existem.

\subsection{Problema 3}

(Russia 2001) Encontre todos os primos $p$ e $q$ tais que $p+q=(p-q)^{3}$.

Solução: Primeiro, note que $(p-q)^{3}=p+q \neq 0$, e com isso $p$ e $q$ são dois primos distintos, donde $\operatorname{mdc}(p, q)=1$ e também temos $m d c(p, p+q)=1$. Agora, note que

$$
\begin{gathered}
p-q \equiv(p-q)+(p+q)(\bmod p+q) \Rightarrow p-q \equiv 2 p(\bmod p+q) \\
\Rightarrow(p-q)^{3} \equiv 8 p^{3}(\bmod p+q) .
\end{gathered}
$$

Por outro lado, o problema forneceu-nos $(p-q)^{3} \equiv 0(\bmod p+q)$, e portanto $8 p^{3} \equiv 0(\bmod p+q) \Rightarrow$ $p+q \mid 8$, donde a última implicação é consequência de $m d c(p, p+q)=1$. Como o único par de primos ímpares cuja soma divide 8 é 5 e 3 , esta é a única solução.

\subsection{Problema 4}

(HMMT 2004) Zach escolheu cinco números do conjunto $\{1,2,3,4,5,6,7\}$. Se ele contasse à Claudia o produto dos números escolhidos, isso não seria suficiente para que ela descobrisse se a soma dos mesmos é par ou ímpar. Qual o produto dos números escolhidos?

Solução: Note que fornecer o produto dos números escolhidos é equivalente a fornecer o produto dos dois números que não foram escolhidos. Os únicos produtos que são obtidos pelo produto de mais de um par de números são: $12(\{3,4\}$ e $\{2,6\})$ e $6(\{1,6\}$ e $\{2,3\})$. No segundo caso, porém, a soma dos dois números não escolhidos é ímpar, donde a soma dos escolhidos também será ímpar. 
Portanto, apenas a primeira opção é válida, e assim o produto dos cinco números escolhidos é igual a

$$
\frac{1 \cdot 2 \cdot 3 \cdots 7}{12}=420
$$

\subsection{Problema 5}

( APMO 2005) Mostre que para todo número real irracional $a$, existem números reais e irracionais $b$ e $b^{\prime}$ tais que $a+b$ e $a b^{\prime}$ são ambos racionais, enquanto $a b$ e $a+b^{\prime}$ são ambos irracionais.

Solução: Seja $a \in \mathbb{R}-\mathbb{Q}$. Se $a^{2}$ é irracional, tome $b=-a$. Então, $a+b=0$ é racional e $a b=-a^{2}$ é irracional. Se $a^{2}$ é racional, tome $b=a^{2}-a$. Daí, $a+b=a^{2}$ é racional e $a b=a^{2}(a-1)$. Assim, $a^{2} \in \mathbb{Q}$ e $a-1 \notin \mathbb{Q}$, donde $a b=a^{1}(a-1)$ é irracional.

Agora, tome $b^{\prime}=\frac{1}{a}$ ou $b^{\prime}=\frac{2}{a}$. Assim $a b^{\prime}=1$ ou 2 , que é racional. Note que

$$
a+b^{\prime}=\frac{a^{2}+1}{a} \quad \text { ou } \quad a+b^{\prime}=\frac{a^{2}+2}{a},
$$

e como,

$$
\frac{a^{2}+2}{a}-\frac{a^{2}+1}{a}=\frac{1}{a} \in \mathbb{R}-\mathbb{Q},
$$

pelo menos um deles é irracional, e escolhemos o $b^{\prime}$ de acordo com isso.

\subsection{Problemas Complementares}

$[1,2,5,7]$

1. (ARML) Encontre o maior divisor de 1001001001 que não excede 10000.

2. (HMTT) Calcule

$$
m d c\left(2002+2,2002^{2}+2,2002^{3}+2, \ldots\right) .
$$

3. (AIME) Calcule a probabilidade de que um divisor positivo de $10^{99}$ escolhido aleatoriamente seja um múltiplo de $10^{88}$.

4. (BW) Seja $a$ um inteiro ímpar. Mostre que $a^{2^{n}}+2^{2^{n}}$ e $a^{2^{m}}+2^{2^{m}}$ são relativamente primos para todos inteiros positivos $n$ e $m$ com $n \neq m$.

5. (RMO) Sejam os números primos $n_{1}<n_{2}<\cdots<n_{31}$. Mostre que se 30 divide $n_{1}^{4}+n_{2}^{4}+\cdots+n_{31}^{4}$, então entre esses números existem três primos consecutivos.

6. (IMO) Considere a sequência $a_{1}, a_{2}, \ldots$ dada por

$$
a_{n}=2^{n}+3^{n}+6^{n}-1,
$$

para todo $n$ inteiro positivo. Determine todos os inteiros positivos que são coprimos com todos os termos dessa sequência. 
7. (AIME) Quantos inteiros positivos múltiplos de 1001 podem ser expressos da forma $10^{j}-10^{i}$, onde $i$ e $j$ são inteiros com $0 \leq i<j \leq 99$ ?

8. (USAMO) Mostre que para todo inteiro positivo $n$, existe um número de $n$ dígitos divisível por $5^{n}$ com todos os seus dígitos ímpares.

9. (ASHME) Para quantos inteiros $N$ entre 1 e 1990 a fração $\frac{N^{2}+7}{N+4}$ não é irredutível?

10. (AIME) Os números da sequência

$$
101,104,109,116, \ldots
$$

são da forma $a_{n}=100+n^{2}$, onde $n=1,2,3, \ldots$. Para cada $n$, seja $d_{n}$ o máximo divisor comum de $a_{n}$ e $a_{n+1}$. Encontre o valor máximo de $d_{n}$ quando $n$ varia sobre todo o conjunto dos inteiros positivos.

11. (OMA) Sejam $p_{1}, p_{2}, \ldots p_{n}$ números primos. Bruno deve escolher $n+1$ inteiros positivos que utilizem apenas esses primos em sua decomposição. Bernardo deve escolher alguns desses números de modo que o produto deles seja um quadrado perfeito. Determine se é possível, para algum $n$, que Bruno escolha seus $n+1$ números de maneira que Bernardo não consiga cumprir seu objetivo.

\section{Abreviações}

[AIME] - American Invitational Mathematics Exam (PC-A 4; PC-TN 3, 7, 10)

[APMO] - Asian Pacific Mathematics Olympiad (P-TN 5)

[ARML] - American Regions Mathematics League (PC-TN 1)

[ASHME] - American High School Mathematics Examination (PC-TN 9)

[BW] - Baltic Way (PC-TN 4)

[CMO] - Canadian Mathematical Olympiad (PC-A 8)

[HMMT] - Harvard-MIT Mathematics Tournament (P-TN 4; PC-TN 2)

[HMO] - Hungarian Mathematical Olympiad (PC-A 11)

[IMO] - International Mathematical Olympiad (PC-A 3; P-TN 1; PC-TN 6)

[IMTT] - International Mathematics Tournament of the Towns (PC-A 9)

[INAMO] - Indonesia Mathematical Olympiad (PC-A 6)

[JBMO] - Junior Balkan Mathematical Olympiad (PC-A 7)

[OBM] - Olimpíada Brasileira de Matemática (P-A 1)

[OCM] - Olimpíada Cearense de Matemática (PC-A 1)

[OMA] - Olimpíada Matemática Argentina (PC-TN 11)

[OMEG] - Olimpíada de Matemática do Estado de Goiás (P-A 3) 
[RMO] - Romanian Mathematical Olympiad (PC-TN 5)

[USAMO] - United States of America Mathematical Olympiad (PC-A 5,10; PC-TN 8)

[VMO] - Vietnamese Mathematical Olympiad (P-A 5)

P-A: Problema de álgebra

PC-A: Problema Complementar de álgebra

P-TN: Problema de Teoria dos Números

PC-TN: Problema Complementar de Teoria dos Números

\section{Referências}

[1] Andreescu, T., Andrica, D. and Feng Z., 104 Number Theory Problems, Birkhauser, Boston, MA, 2007.

[2] F. E. Brochero Martinez, C. G. Moreira, N. C. Saldanha, E. Tengan Teoria dos Números: um passeio com primos e outros números familiares pelo mundo inteiro, Projeto Euclides, IMPA, 2010.

[3] Carneiro, E., Campos, O. e Paiva, F. Olimpíadas Cearenses de Matemáatica 1981-2005 (Níveis Júnior e Senior), Ed. Realce, 2005.

[4] Chau, L. H. and Khoi, L. H. Selected Problems of the Vietnamese Mathematical Olympiad (1962-2009), World Scientific, Danvers MA, 2010.

[5] Feitosa, S. B. , Holanda, B., Lima, Y. e Magalhães, C. T. Treinamento Cone Sul 2008. Fortaleza, Ed. Realce, 2010.

[6] Fomin, D. and Kirichenko, A. Leningrad Mathematical Olympiads 1987-1991, MathPro Press, Westford, MA, 1994.

[7] Fomin, D., Genkin, S. and Itenberg, I. Mathematical Circles, Mathematical Words, Vol. 7, American Mathematical Society, Boston, MA, 1966.

[8] Niven, I., Zuckerman, H. S. and Montgomery, H. L. An Introduction to the Theory of Numbers.

Ana P. Chaves

Universidade Federal de Goiás Instituto de Matemática e Estatística

Goiânia/GO <apchaves@ufg.br> 